\title{
Microbial Biofilms in Biliary and Pancreatic Stents
}

\author{
Chetana Vaishnavi ${ }^{1}$, Nikhil Bush ${ }^{2}$, Rakesh Kochhar ${ }^{3}$
}

\begin{abstract}
Biofilms are formed by unicellular bacteria residing on a scaffold of exopolymeric substances and impart survival advantage to microbial communities from adverse environmental conditions. The formation of biofilms is an intricately ordered stepwise process. Biofilms can form on many medical implants where the body fluids supply adequate organic nutrients for the most favorable bacterial growth. The hydrophobicity of the surface influences the strength of adhesion and microorganisms are more likely to attach to non-polar hydrophobic surfaces, which make up stents. In general, the stent diameter, duration of stenting, contour and material determine the susceptibility of stents to biofilmrelated occlusion. Occlusion of stents by microcolonies can result in increased rates of complications and therapeutic failure leading to an increased need for repeat procedures and hence cost of treatment. Despite several studies explicating the range of organisms involved in the formation of biofilms, the factors related to their formation in plastic stents have not been adequately elaborated. Biofilms cannot be detected by standard microbial culture techniques. Molecular diagnostics based on nucleic acid amplification techniques are the mainstay for detection and identification. The formation of microbial biofilms in bio prosthesis related to gastroenterology is an under studied arena. In this minireview, we aim to discuss the formation of biofilms in biliary and pancreatic stents used for the management of biliary and pancreatic ductal obstructions to provide clinicians with information to optimize the safety and efficiency of stents.

Keywords: Biliary stents, Biofilms, Microbial colonization, Occlusion, Pancreatic stents.

Journal of Gastrointestinal Infections (2020): 10.5005/jp-journals-10068-3037
\end{abstract}

\section{INTRODUCTION}

A biofilm is defined as a conglomeration of microbial communities embedded within an extracellular matrix attached to man-made or natural surfaces. ${ }^{1}$ Biofilms are produced when unicellular bacteria begin to reside on a scaffold of exopolymeric substances largely comprised of proteins and polysaccharides secreted by the resident microorganisms themselves, often being separated by networks of open water channels. ${ }^{2}$ About $5-35 \%$ of the biofilm quantity is formed by the microorganisms while the remainder constitutes the extracellular matrix. ${ }^{3}$ Biofilms impart survival advantage to microbial communities from adverse environmental conditions like biological and chemical antibacterial agents due to the inactivation of these agents by extracellular polymers., ${ }^{4,5}$ Furthermore, biofilm formation has been postulated as a phylogenetic evolution of the bacteria in the emergence of antibiotic-resistant strains as a result of phenotypic changes in the residing bacteria. ${ }^{6,7}$

Biofilms can form on many medical implants, such as, catheters and stents where the body fluids supply adequate organic nutrients for the most favorable bacterial growth. ${ }^{8}$ The sphincter of Oddi serves as a gatekeeper to the pancreatic and biliary ducts against microbes. This natural barrier gets broken upon placing a stent across it, resulting in a low resistance pathway for the colonization of intestinal microbes. Occlusion of stents by microcolonies can result in an increased rates of complications and therapeutic failure leading to increased need for repeat procedures and hence cost of treatment. Previous studies have estimated that about $65 \%$ of all device and non-device associated infections are related to bacterial biofilms. ${ }^{9}$ The resident gastrointestinal microbial biofilms have been implicated in diseases, such as, inflammatory bowel disease, colon cancer, gastric cancer, and irritable bowel syndrome. ${ }^{10}$ Despite the several studies explicating the range of organisms involved in the formation of biofilms, the factors related to their formation in plastic stents and their clinical implications need to
${ }^{1-3}$ Department of Gastroenterology, Postgraduate Institute of Medical Education and Research, Chandigarh, India

Corresponding author: Chetana Vaishnavi, Department of Gastroenterology, Postgraduate Institute of Medical Education and Research, Chandigarh, India, e-mail: cvaishnavi@rediffmail.com

How to cite this article: Vaishnavi C, Bush N, Kochhar R. Microbial Biofilms in Biliary and Pancreatic Stents. J Gastrointest Infect 2020;10(1):11-15.

Source of support: Nil

Conflict of interest: None

be adequately elaborated before any steps can be taken for their successful prevention. ${ }^{1,4,11}$ In this mini-review, we aim to discuss the formation of biofilms in biliary and pancreatic stents used for the management of biliary and pancreatic ductal obstructions to provide clinicians with information to optimize the safety and efficiency of stents.

\section{Steps in Biofilm Formation}

Formation of biofilms is an intricately ordered stepwise process involving the following steps: initial surface contact, next microcolony formation, maturation of architecture of the biofilm and lastly detachment/dispersion of the biofilm. ${ }^{12}$ The process of attachment to the surface of animate or inanimate objects occurs through pili or flagella and other physical forces like Van der Waal's forces and the attractive or repulsive electrostatic interactions. The hydrophobicity of the surface also influences the strength of adhesion and microorganisms are more likely to attach to non-polar hydrophobic surfaces of plastics (e.g. Teflon) which make up stents rather than hydrophilic polar surfaces. ${ }^{13}$

Following the stabilization of the attachment, microcolonies are formed by multiplication of microbial cells within the extracellular matrix. These colonies form a coordinated symbiotic 
relationship among each other in multiple aspects including the exchange of substrates and distribution and excretion of metabolic products. ${ }^{14}$ Maturation of these colonies is marked by the expression of genes that cause the production of the extracellular matrix and signal molecules known as auto inducers which facilitate quorum sensing-a cell-to-cell intra- and intercolonial communication system. The matrix serves as the scaffold or backbone of the biofilm which is further laden with interstitial voids filled with water that serve as circulatory channels for nutrients and waste products. Subsequently, the bacteria produce saccharolytic enzymes or express genes upregulating fimbriae/pili formation thereby facilitating detachment and transfer to a new site. ${ }^{2}$

\section{Stent Related Risk Factors for Biofilm Formation}

In general, the stent diameter, duration of stenting, contour, and material determine the susceptibility of stents to biofilm-related occlusion. Stents with smaller diameters have a higher predisposition for biofilm formation. The bile flow dynamics and stent occlusion are governed by the diameter of the biliary stents and an increase of $0.2 \mathrm{~mm}$ diameter in the inner stent leads to a $300 \%$ increase in the bile flow. ${ }^{15}$ The large diameter of the metallic stent is one of its major advantages. ${ }^{16}$ However at best plastic stent of a maximum of 11.5 Fr diameter can be placed endoscopically ${ }^{17}$ and therefore stents up to $10 \mathrm{Fr}$ size are generally used. Rates of bacterial colonization increase linearly with prolonged durations of stenting, progressively increasing after 3 months with median patency of $4-5$ months for 10 Fr plastic stents. ${ }^{18}$ Therefore, plastic biliary stents should be changed by 3-6 months to avert the formation of biofilms. Irregularities in the surface and hydrophobicity of the stent material can also increase the adherence of bacteria and thereby increase biofilm formation. Additionally bile immunoglobulin-bacteria complex, glycoprotein, and mucin can also affect bacterial adherence. ${ }^{19}$

\section{Detection and Characterization of Biofilms}

The characterization of biofilms is based on a range of techniques, which include direct methods like plate count method, flow cytometry, fluorescent, and confocal scanning microscopy. Indirect quantification methods include crystal violet assay, ATP bioluminescence, and quartz crystal microbalance techniques. These techniques are fraught with technical limitations and are less cost-effective. Culture-based methods are the most accurate and reliable for characterizing and identifying biofilms. ${ }^{20}$ However, biofilms cannot be detected by standard microbial culture techniques. Molecular diagnostics based on nucleic acid amplification techniques are the mainstay for detection and identification. Diagnosis, treatment, and prevention of biofilm infections require different kinds of strategies apart from those used against acute infections. For identification of the bacteria by molecular methods, the encrusted biofilm inside the stent is scraped and cultured in liquid media under aerobic and anaerobic conditions. The microbial DNA is then removed from the culture media by the phenol-chloroform method. Further characterization of bacteria is done by polymerase chain reaction (PCR) using the $16 \mathrm{~S}$ rRNA gene-specific primers for determination of the DNA sequence for commonly known bacteria using standard strains. The generated amplicons are further stained with ethidium bromide and evaluated with a database of known sequences. Unknown bacteria involved in biofilm formation in the stents are molecularly identified using universal primers with a touchdown PCR used to increase the specificity of the reaction. The selected Density Gradient Gel Electrophoresis bands are excised from the gels and the amplified PCR products are sequenced using bands, which are dissimilar from the usually known bands and are compared with GenBank data base using standard nucleotide blast search tools. ${ }^{1,21}$

\section{Organisms in Biliary and Pancreatic Biofilms \\ Biliary Biofilms}

The role of multiple species of microorganisms in the formation of biofilms responsible for the occlusion of stents is well known. Biofilms maybe of endogenous origin from the native gut microbiota or can be acquired exogenously from contaminated prosthesis or infusates. Both aerobic and anaerobic bacterial species encompassing gram-negative as well as gram-positive organisms are included among the microorganisms isolated from occluded biliary stents. $^{22}$

Enterococcus sp. is an important aerobic pathogen in stentrelated infections and their growth within the biofilms is a vital clinical concern because of antibiotic resistance. ${ }^{8}$ Enterococcus carrying aggregation substance genes and slime-producing strains have a selective advantage in endoprosthesis colonization thereby accounting for their higher rates of prevalence in occluded stents. ${ }^{23}$ Apart from this, the most frequent aerobic bacteria isolated from biliary sludge are Escherichia coli and Klebsiella although Clostridium species are the predominant anaerobes. ${ }^{11,24}$ Leung et al. ${ }^{25}$ identified 19 anaerobes (Clostridium perfringens 13, C. bifermentans 4, and Bacteroides fragilis 2) from 16 unblocked stents retrieved from patients who received antibiotic prophylaxis and 16 gram-positive aerobes (Enterococcus and Bacillus); but no gram-negative aerobes were isolated from the stents. Schneider et al. ${ }^{11}$ also reported anaerobes as prominent inhabitants of biofilms in biliary stents. Vaishnavi et al. ${ }^{1}$ reported polybacterial consortia in $90 \%$ of the biliary stents with the most commonly identified species being Pseudomonas, Citrobacter, and Klebsiella; with a relatively small proportion of isolates belonging to Staphylococcus, Serratia, Streptococcus, Enterococcus, Proteus and Enterobacter species. Figure 1 depicts a double pig tail occluded biliary stent which was removed after 4 months of placement. No difference in species composition was noted between stents retrieved from patients with or without cholangitis despite that patients with cholangitis tend to have higher rates of complete occlusion and biofilm formation. ${ }^{1}$ However, occluded stents showed a higher prevalence of colonization by gram-positive organisms like Staphylococcus, Streptococcus, and Enterococcus. ${ }^{1,11}$ Recently Kong and $\mathrm{Xie}^{26}$ analyzed the bacterial species in occluded plastic stents retrieved from 60 elderly patients with malignant biliary obstruction and identified a total of 93 pathogenic bacteria of which 61 cases were gram-negative bacilli (65.6\%), 30 cases were gram-positive

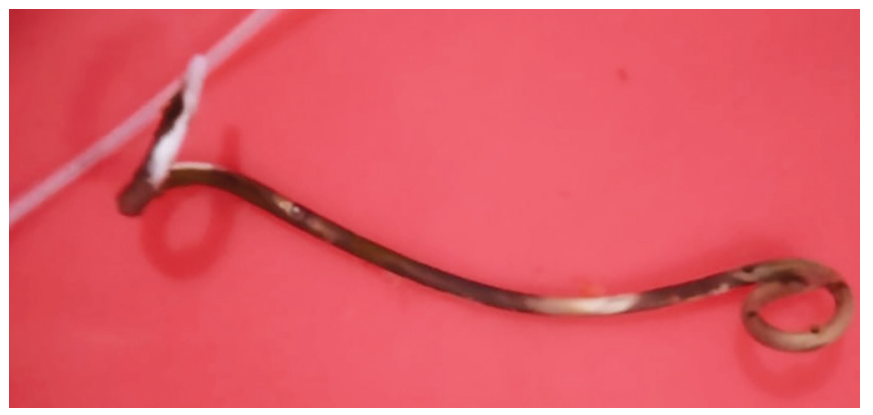

Fig. 1: Double pig tail biliary stent (7 Fr) with biofilm retrieved after 4 months of placement 
cocci $(32.2 \%)$, and 2 were fungi $(2.2 \%)$ cases. The most common bacteria identified were E. coli (22.6\%), Enterococcus faecium (16.1\%), Klebsiella pneumoniae (14.0\%), Pseudomonas aeruginosa (11.8\%), and Enterococcus faecalis (10.8\%).

\section{Pancreatic Biofilms}

Kozarek et al. ${ }^{27}$ reported contamination of the pancreatic duct by gut flora in $100 \%$ of 36 patients with pancreatic stents. Though gut appears to be the main source of bacteria for stent occlusion, at times gram-positive bacteria, such as, Staphylococcus aureus, Enterococcus, and anaerobes can also be found. Hill et al. $^{28}$ reported bacterial growth composed of bacteria, such as, Klebsiella, Escherichia, Enterobacter, and Enterococcus in 28/47 retrieved pancreatic stents, with a strong correlation between the duration of indwelling stents and the bacterial growth. The species diversity of pancreatic stent biofilms was further testified in a study that characterized more than 246 resident species. ${ }^{11}$ Similarly Schneider et al. ${ }^{29}$ reported 246 microorganisms in 39/40 of the pancreatic stents among which gram-positive Enterococcus sp. was predominantly present in 27 of them.

Vaishnavi et al. ${ }^{30}$ identified polymicrobial biofilms in $95.8 \%$ of the 24 retrieved occluded pancreatic stents. The biofilm comprised of Pseudomonas ( $n=8)$, Staphylococcus $(n=8)$, Serratia $(n=5)$, Aeromonas $(n=4)$, Proteus $(n=4)$, Klebsiella $(n=4)$, E. coli $(n=4)$, Enterococcus $(n=4)$, Streptococcus $(n=4)$, Citrobacter $(n=3)$, Bacillus $(n=2)$, Enterobacter $(n=1)$, Vibrio $(n=1)$, and Clostridium ( $n=1$ ) as identified by PCR and sequencing. ${ }^{1}$ Anaerobes are known to be important in biofilm formation, ${ }^{11}$ but Clostridium sp. was found in only a single pancreatic stent with an indwelling time of 150 days. ${ }^{30}$

\section{Impact of Biofilms in Relation to Biliary and Pancreatic Diseases}

The impact of biofilms in gastroenterology and its implications have been under studied. It has been estimated that biofilms are associated with $65 \%$ of nosocomial infections and that treatment of these infections is very difficult and leads to increased health care expenditure. $^{31,32}$ Biofilm formation on stents might have an impact on the underlying disease process. Clogging of stents with secretions and subsequent microbial colonization can lead to stent dysfunction. The course of formation of biofilm is a very complex and gradual process, which starts about 3 months after stent placement and is directly proportional to the dwelling time. ${ }^{11,33}$

Biliary plastic stents were introduced in 1979 and stenting through the endoscope is a non-surgical approach for relieving obstructions in the biliary structure. The sphincter of Oddi is located at the junction of the common bile duct, main pancreatic duct, and the duodenum is a natural microbial barrier to biliary contamination. ${ }^{34}$ When a biliary stent is introduced across it, the sphincter gets breached and leads to reflux of the duodenal material, and ascending bacterial colonization occurs leading to biofilm formation. Stent failure secondary to occlusion can lead to cholangitis, which is a medical emergency requiring timely biliary drainage. Another implication of biofilms in cholangitis is the development of mechanisms for microbial persistence and drug resistance making the existing management strategies ineffective. ${ }^{35}$ One of these is the exopolysaccharide matrix scaffold that provides adequate defense from natural surfactants and antibacterial agents including bile and inflammatory cells. ${ }^{2}$

Similarly, several pancreatic diseases can be managed by endoscopic placement of stents that can help decrease intraductal pressure, restore luminal patency in pancreatic strictures, and bypass obstructing stones. Pancreatic duct stenting is carried out in chronic pancreatitis with major duct strictures to relieve pain or in a setting of duct disruption and persistent pancreatic collections. ${ }^{36}$ Blockage and consequent stent dysfunction can result in acute deterioration of the underlying pathology as well as serve as a microbial stairway for infection of pancreatic collections.

Guaglianone et al. ${ }^{37}$ studied the tridimensional arrangement of the microbial biofilm formed inside clogged biliary stent to identify the microbial species implicated in the obstruction process. Scanning electron microscopy studies revealed that sludge present in the stent lumen consisted of a rich and mixed aerobic and anaerobic microbial flora interspersed with a large amount of amorphous material containing dietary fibers, cholesterol crystals, and other bile salts precipitates. ${ }^{38}$ Biofilm matrix comprise of proteins, polysaccharides, nucleic acids, and lipids. ${ }^{39}$ Vaishnavi et al. ${ }^{30}$ reported that the majority of the pancreatic stents harbored multiple microorganisms in their biofilms, and those from patients aged $>50$ years showed significant deposition of protein, which is a key element in biofilm formation, thereby suggesting that advanced age is a risk factor for the deposition of biofilm proteins. ${ }^{30}$

A direct consequence of stent occlusion related to biofilm formation is the need for repeated interventions and the added risk of complications and costs. This is thus a major limiting factor for the use of plastic stents in these circumstances and if used it must be restricted to the standard duration of a maximum of 3 months. A more durable alternative to plastic stents is metallic stents with longer shelf lives of up to 6 months. There is a lack of data on biofilm characteristics of metallic stents. However, there have been reports of biofilm-related stent clogging in silicone covered self-expanding metallic stents. ${ }^{40}$ Therefore, preventing biofilm formation in stents is a major challenge and various techniques have been tried in the past. Numerous studies have evaluated the antibacterial role of silver nanoparticles in plastic and metallic stents, a few of which have implicated prolonged stent patency, but the overall clinical efficacy has not been completely evaluated as of yet. ${ }^{41,42}$ An upcoming role of biodegradable stents as an alternative to plastic and metallic stents is being received with enthusiasm. Technological interventions are being undertaken in the field of nanotechnology for the development of innovative antimicrobial materials. These agents are utilized to alter the biodegradable polymeric materials from which they are gradually released and thwart the formation of a biofilm on the surface of the polymeric material. ${ }^{43}$

\section{ConCLUSION}

The formation of microbial biofilms in bio prosthesis related to gastroenterology is an under studied arena. Biliary and pancreatic stents are prone to microbial colonization and biofilm formation, which is an important cause of stent occlusion, thereby decreasing the shelf life of stents and hence increasing the need for repeated procedures. Knowledge of the pathophysiology of formation, biochemical composition and identification of patient and stentrelated factors determining biofilm formation, could help in developing preventive strategies against complications related to stent occlusion. There is hence a need to harness better materials with antibacterial properties in stent manufacturing and the role of silver nanoparticles in this regards needs to be further validated in the clinical context. 


\section{References}

1. Vaishnavi C, Samanta J, Kochhar R. Characterization of biofilms in biliary stents and potential factors involved in occlusion. World $\mathrm{J}$ Gastroenterol 2018 7;24(1):112-123. DOI: 10.3748/wjg.v24.i1.112.

2. Costerton JW, Stewart PS, Greenberg EP. Bacterial biofilms: a common cause of persistent infections. Science 1999;284(5418):1318-1322. DOI: 10.1126/science.284.5418.1318.

3. Sun D, Accavitti MA, Bryers JD. Inhibition of biofilm formation by monoclonal antibodies against Staphylococcus epidermidis RP62A accumulation-associated protein. Clin Diagn Lab Immunol 2005;12(1):93-100. DOI: 10.1128/CDLI.12.1.93-100.2005.

4. Lübbert C, Wendt K, Feisthammel J, et al. Epidemiology and resistance patterns of bacterial and fungal colonization of biliary plastic stents: a prospective cohort study. PLoS One 2016;11(5):e0155479. DOI: 10.1371/ journal.pone.0155479.

5. Kumar A, Alam A, Rani M, et al. Biofilms: survival and defense strategy for pathogens. Int J Med Microbiol 2017;307(8):481-489. DOI: 10.1016/j.ijmm.2017.09.016.

6. Jamal M, Ahmad W, Andleeb S, et al. Bacterial biofilm and associated infections. J Chin Med Assoc 2018;81(1):7-11. DOI: 10.1016/j. jcma.2017.07.012.

7. Yan J, Bassler BL. Surviving as a community: antibiotic tolerance and persistence in bacterial biofilms. Cell Host Microbe 2019;26(1):15-21. DOI: 10.1016/j.chom.2019.06.002.

8. Khatoon Z, McTiernan CD, Suuronen EJ, et al. Bacterial biofilm formation on implantable devices and approaches to its treatment and prevention. Heliyon 2018;4(12)e01067. DOI: 10.1016/j. heliyon.2018.e01067.

9. Lewis K. Riddle of biofilm resistance. Antimicrob Agents Chemother 2001;45(4):999-1007. DOI: 10.1128/AAC.45.4.999-1007.2001.

10. von Rosenvinge EC, O'May GA, Macfarlane $S$, et al. Microbial biofilms and gastrointestinal diseases. Pathog Dis 2013;67:25-38. DOI: 10.1111/2049-632X.12020.

11. Schneider J, Hapfelmeier A, Fremd J, et al. Biliary endoprosthesis: a prospective analysis of bacterial colonization and risk factors for sludge formation. PLoS One 2014;9(10):e110112. DOI: 10.1371/journal. pone. 0110112 .

12. Sutherland IW. The biofilm matrix - an immobilized but dynamic microbial environment. Trends Microbiol 2001;9(5):222-227. DOI: 10.1016/s0966-842x(01)02012-1.

13. Vasudevan R. Biofilms: microbial cities of scientific significance. J Microbiol Exp 2014;1(3):00014. DOI: 10.15406/jmen.2014.01. 00014.

14. Otto M.Staphylococcalinfections:mechanisms of biofilm maturation and detachment as critical determinants of pathogenicity. Annu Rev Med 2013;64:175-188. DOI: 10.1146/annurev-med-042711140023.

15. Scheeres $D, O$ 'Brien W, Ponsky L, et al. Endoscopic stent configuration and bile flow rates in a variable diameter bile duct model. Surg Endosc 1990;4(2):91-93. DOI: 10.1007/BF00591267.

16. Soderlund C, Linder S. Covered metal versus plastic stents for malignant common bile duct stenosis: a prospective, randomized, controlled trial. Gastrointest Endosc 2006;63(7):986-995. DOI: 10.1016/j.gie.2005.11.052.

17. Pfau PR, Pleskow DK, Banerjee $S$, et al. Pancreatic and biliary stents. Gastrointest Endosc 2013;77(3):319-327. DOI: 10.1016/j. gie.2012.09.026.

18. Dumonceau J-M, Devière $J$, Delhaye $M$, et al. Plastic and metal stents for postoperative benign bile duct strictures: the best and the worst. Gastrointest Endosc 1998;47(1):8-17. DOI: 10.1016/s00165107(98)70292-5.

19. Sicard J-F, Le Bihan G, Vogeleer $P$, Jacques $M$, Harel J. Interactions of intestinal bacteria with components of the intestinal mucus. Front Cell Infect Microbiol 2017;7:387. DOI: 10.3389/fcimb.2017.00387.

20. Wilson C, Lukowicz R, Merchant S, et al. Quantitative and qualitative assessment methods for biofilm growth: a mini-review. Res Rev J Eng Technol 2017;6(4):1-42.
21. Sanchez CJ, Mende K, Beckius ML, et al. Biofilm formation by clinical isolates and the implications in chronic infections. BMC Infect Dis 2013;13:47. DOI: 10.1186/1471-2334-13-47.

22. Sung, JJY. Bacterial biofilm and clogging of biliary stents. J Ind Microbiol 1995;15(3):152-155. DOI: 10.1007/BF01569819.

23. Johnson JR, Clabots $\mathrm{C}$, Hirt $\mathrm{H}$, et al. Enterococcal aggregation substance and binding substance are not major contributors to urinary tract colonization by Enterococcus faecalis in a mouse model of ascending unobstructed urinary tract infection. Infect Immun 2004;72(4):2445-2448. DOI: 10.1128/iai.72.4.2445-2448.2004.

24. Masák J, Čejková A, Schreiberová O, et al. Pseudomonas biofilms: possibilities of their control. FEMS Microbiol Ecol 2014;89(1):1-14. DOI: 10.1111/1574-6941.12344.

25. Leung JW, Liu Y-L, Chan RC, et al. Early attachment of anaerobic bacteria may play an important role in biliary stent blockage. Gastrointest Endosc 2000;52:725-729. DOI: 10.1067/mge.2000.108964.

26. Kong G, Xie X. Analysis of bacteriology and antibiotic resistance of plastic stent obstruction in elderly patients with malignant biliary obstruction. Adv. Clin. Med 2020;10(7):1298-1305. DOI: 10.12677/ ACM.2020.107197.

27. Kozarek R, Hovde, O, Attia F, et al. Do pancreatic duct stents cause or prevent pancreatic sepsis? Gastrointest Endosc 2003;58(4):505-509. DOI: 10.1067/s0016-5107(03)01891-1.

28. Hill SK, Bhalla C, Thomson A. Risk of bacterial colonization of pancreatic stents used in endoscopic retrograde cholangiopancreatography. J Clin Gastroenterol 2012;46(4):324-327. DOI: 10.1097/ MCG.0b013e31823712cc.

29. Schneider J, Schenk P, Obermeier A, et al. Microbial colonization of pancreatic duct stents: a prospective analysis. Pancreas 2015;44(5): 786-790. DOI: 10.1097/MPA.0000000000000332.

30. Vaishnavi C, Gupta PK, Sharma M, et al. Quantification of major constituents of biofilms in occluded pancreatic stents. J Med. Microbiol 2018;67(9):1225-1231. DOI: 10.1099/jmm.0.000805.

31. Archibald LK, Gaynes RP. Hospital-acquired infections in the United States. The importance of interhospital comparisons. Infect Dis Clin North Am 1997;11:245-255. DOI: 10.1016/s08915520(05)70354-8.

32. Lebeaux D, Ghigo J-M, Beloin C. Biofilm-related infections: bridging the gap between clinical management and fundamental aspects of recalcitrance toward antibiotics. Microbiol Mol Biol Rev 2014;78(3):510-543. DOI: 10.1128/MMBR.00013-14.

33. Donlan RM. Biofilms: microbial life on surfaces. Emerg Infect Dis 2002;8(9):881-890. DOI: 10.3201/eid0809.020063.

34. Seetharam P, Rodrigues $G$. Sphincter of Oddi and its dysfunction. Saudi J Gastroenterol 2008;14(1):1-6. DOI: 10.4103/1319-3767.37793.

35. Mah TF, O'Toole GA. Mechanisms of biofilm resistance to antimicrobial agents. Trends Microbiol 2001;9(1):34-39. DOI: 10.1016/s0966842x(00)01913-2.

36. Mangiavillano B, Pagano N, Baron TH, et al. Outcome of stenting in biliary and pancreatic benign and malignant diseases: a comprehensive review. World J Gastroenterol 2015;21(30):9038-9054. DOI: 10.3748/wjg.v21.i30.9038.

37. Guaglianone E, Cardines R, Mastrantonio P, et al. Role of multispecies microbial biofilms in the occlusion of biliary stents. Microb Ecol Health Dis 2008;20(4):207-209. DOI: 10.1080/08910600802408145.

38. Weickert U, Zimmerling S, Eickhoff A, et al. A comparative scanning electron microscopic study of biliary and pancreatic stents. Z Gastroenterol 2009;47(4):347-350. DOI 10.1055/s-2008-1027926.

39. Pereira, AL, Silva TN, Gomes ACMM, et al. Diarrhea-associated biofilm formed by enteroaggregative Escherichia coli and aggregative Citrobacter freundii: a consortium mediated by putative F pili. BMC Microbiol 2010;10:57. DOI: 10.1186/1471-2180-10-57.

40. Masayuki K, Yamashita Y, Tanaka K, et al. Covered self-expandable metal stents with an anti-migration system improve patency duration without increased complications compared with uncovered stents for distal biliary obstruction caused by pancreatic carcinoma: a randomized multicenter trial. Am J Gastroenterol 2013;108(11):17131722. DOI: $10.1038 / a j g .2013 .305$. 
41. Lee TH, Jang BS, Jung MK, et al. Fabrication of a silver particleintegrated silicone polymer-covered metal stent against sludge and biofilm formation and stent-induced tissue inflammation. Sci Rep 2016;6:35446. DOI: 10.1038/srep35446.

42. Lee TH, Jung MK, Kim T-K, et al. Safety and efficacy of a metal stent covered with a silicone membrane containing integrated silver particles in preventing biofilm and sludge formation in endoscopic drainage of malignant biliary obstruction: a phase 2 pilot study. Gastrointest Endosc 2019;90(4):663-672. DOI: 10.1016/j.gie.2019.06.007.

43. Wang L, Hu C, Shao L. The antimicrobial activity of nanoparticles: present situation and prospects for the future. Int J Nanomedicine 2017;12:1227-1249. DOI: 10.2147/IJN.S121956. 